Journal of Engineering and Applied Sciences 14 (12): 4109-4115, 2019

ISSN: 1816-949X

(C) Medwell Journals, 2019

\title{
Spatio-Temporal Query Processing Operators in Sensor Networks
}

\author{
Jeong-Joon Kim \\ Department of Computer Science and Engineering, Korea Polytechnic University, \\ 15073 Gyeonggi-do, Siheung-si, South Korea
}

\begin{abstract}
As technologies related to sensor network are currently emerging and the use of GeoSensor is increasing along with the development of IoT technology, spatial query processing systems to efficiently process spatial sensor data are being actively studied. However, existing spatial query processing systems do not support a spatial-temporal data type and a spatial-temporal operator for processing spatial-temporal sensor data. Therefore, they are inadequate for processing spatial-temporal sensor data like GeoSensor. Accordingly, this study developed a spatial-temporal query processing operator for efficient spatial-temporal query processing of spatial-temporal sensor data in a sensor network.
\end{abstract}

$\underline{\text { Key words: Sensor networks, sensor query processing, data type, operator, development, GeoSensor }}$

\section{INTRODUCTION}

In addition to the development of IoT (Internet of Things) technology which can connect a sensor to all of the recent circum-stances and manage various information by connecting it to an internet it is also possible to use a sensor, RFID, CCTV, Wireless communication technologies such as wireless LAN (WiFi), Bluetooth (Bluetooth) and Zigbee have been developed rapidly and are being used for military, medical, weather, various researches have been carried out to utilize sensor network related technology in some fields of application such as wireless sensor networks (D'Ulizia et al., 2012; Ale, 2012).

The sensor network consists of a number of sensor nodes. Sensor nodes consist of sensing, processing, storage and telecom modules and each sensor node is highly limited to hardware and software. Above all, energy capacity is small and data transfer is critical due to data transfer. Thus, in the sensor network, there is a study of the characteristics of the sensor nodes and a study to better address various queries with low compute capacities and small power capacity (Kim et al., 2009).

Therefore, this study has developed a space operator to efficiently provide query processing for multi-dimensional data in these sensors. In this thesis, the operator implements the "Simple Features Recommendation for SQL" standards set forth in the International Space Information Standardization Organization for interoperability (Anonymous, 201 1a, b). The operators of this study consist of space time relation operators, time operators, relation operators and tracking operators.

\section{Literature review}

STT (working with Spatio-Temporal data Type): In order to deal with spatial-temporal inquiry in relational database systems, STT operators presented new concept operators. This improves time to distinguish between the hours by dividing it into the right (left) and later (right). Table 1 shows a typical example, divided by and after the time operator in STT (Kim et al., 2013).

As shown in Table 1, STT is divided into STT and STT and STT and STT are divided into the same format. Also, the space between the left and right side of the

Table 1: STT operator

Operators
STT_overlaps (time A,
B): Boolean
STT_left_overlaps (time A,
B): Boolean
STT_right_overlaps (time A,
B): Boolean
STT_covers (time A,
B): Boolean
STT_left_covers (time A,
B): Boolean
STT_right_covers (time A,
B): Boolean
STT_covered (time A,
B): Boolean
STT_left_covered (time A,
B): Boolean
STT_right_covered (time A,
B): Boolean



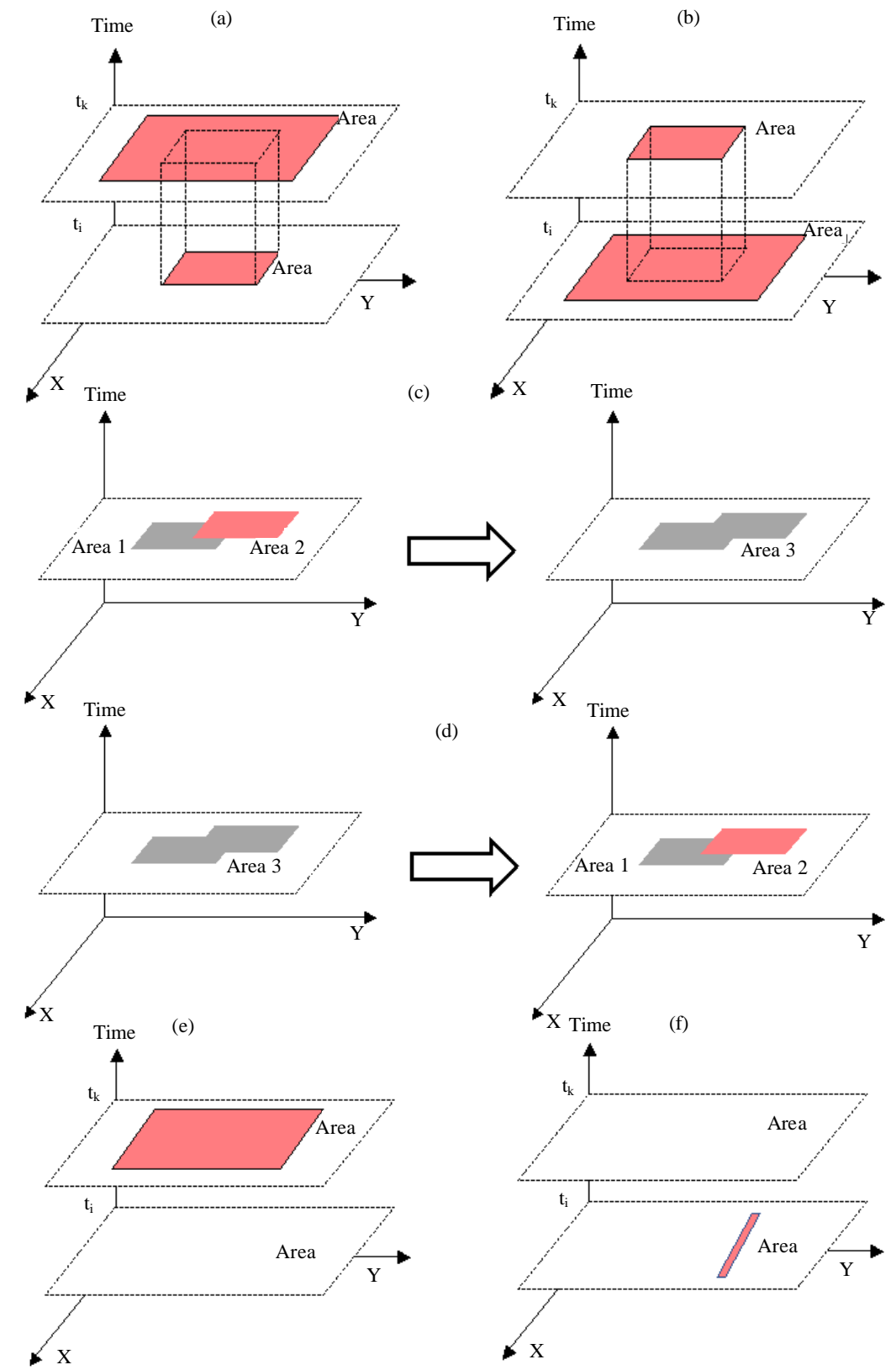

Fig. 1: Example of spatio-temporal operator of GeoPQL: a) Geo_growing; b) Geo_shrinking; c) Geo_merging; d) Geo_splitting; e) Geo_appearing and f) Geo_disappearing

space operator was reflected in the left and right. The prefixes such as left and right, depend on whether the second parameter (T2) falls in the range of the second parameter (T1) or the right range, depending on the first parameter (T1).

In STT, the STT and STT operators can extract the same results from the two parameter sequences, so, one operator is not required. However, it is necessary to divide the function by dividing it into the right (left) and later (right).

\section{MATERIALS AND METHODS}

Moving GeoPQL (a pictorial language towards spatiotemporal queries): In order to handle spatial and spatial inquiry in the mobile trace database system, the moving arrow presented a new concept operator. By Kim et al. (2015) moving forward, moving new space operators into a variety of spatial and spatial questions has resulted in moving forward. Figure 1 shows examples of the spatial operator space provided by the moving segments. 
As shown in Fig. 1, growing operator returns whether certain zones are extended over certain zones at tk time and the shrinking operator returns whether certain areas are smaller than certain zones at off-peak times. The merging operator returns two specific zones at the same time in the same time zone and the splitting operator returns to a specific area divided by the segmented area if a particular area is split. The appearing operator returns whether certain zones are present at ti time and disappearing operator returns, the Geo operator has certain zones in the ti time and the tk operator returns whether certain areas have disappeared at tk time.

In moving GeoPQL and shrinking operators, operators are not required to assume that a particular area has been extended or shrink compared to before, since, the sensor nodes are not expanded or shrinking, since, the sensor nodes are not expanded or shrinking. Whereas the operators of appearing and disappearing operators are required to identify that a particular area exists and appears to disappear while the sensor node appears to be missing or added, these operators are required to disappear or disappear while the sensor node appears to disappear or disappear.

Operator design: This section details temporal relationship operators, time analysis operators, temporal space operators, temporal space analysis operators, temporal space analysis operators and temporal space tracking operators who configure temporal space operators.

Time relation operator: The time relationship operators processing module provides a time relationship operator to support the processing of time operations on the sensor node. The operator is divided into the time stamp (timestamp) operator and interval operator and returns true or false returns for the operation. Table 2 shows the types of time stamp/interval time relationships.

As shown in Table 2, the time stamp/interval time relation operator receives spatio-temporal object $\mathrm{A}$ and time $\mathrm{B}$ as input values, performs time calculation and returns true or false. For example, tDisjoint operators, tLeftDisjoint operators and operators of tRightDisjoint operators provide tLeftDisjoint and tRightDisjoint operators in order to distinguish between time and time in order to distinguish between hours and hours.

Time analysis operator: The time analytics operator processing module provides a time analysis operator to support the processing of time operations on the sensor node. This operator is divided into time stamp operators and interval operators and returns time data types for the operation. Table 3 shows the type of time stamp/interval time analysis operator.

As shown in Table 3, the time stamp time analyzer takes two times $\mathrm{A}$ and $\mathrm{B}$ inputs and performs a time operation to return the time data type. For example, tUnion, a time analysis operator, accepts two times interval (stTime, edTime) and returns the time span to the time data type.

Space time operator: Space time relationship operator processing modules extend OGC's "Simple Features Specification for SQL" standard specifications to support processing of space and spatial processing in the sensor node, thereby providing temporal and spatial operators. The operator is divided into time stamp operators and interval operators and returns true or false returns for the operation. Table 4 shows the types of time stamp/interval space-time relationships.

As shown in Table 4, the time stamp/interval spatio-temporal relational operator receives two spatio-temporal objects $\mathrm{A}$ and $\mathrm{B}$ as input values, performs spatio-temporal operations and returns true or false. For example, among stContains operators, stLeftContains operators, stContains operators and stRightContains operators provide stLeftContains and stRightContains operators to classify and interact with specific space objects in order to distinguish between time and space objects.

Space time analysis operator processing module: The space time analytic operator processing module provides space time analysis operators by expanding the OGC's "Simple Features Specification for SQL" standard to support processing of space and spatial processing on the sensor nodes. This operator is divided into time stamp operators and interval operators and returns space time data types for the operation. Table 5 shows the types of time stamp/interval space-time analysis operators.

As shown in Table 5, the time stamp and time interval analysis operators enter two spatial space time data types by typing two times interval objects $\mathrm{A}$ and $\mathrm{B}$ and then return space data type. For example, stIntersection of spatio-temporal analysis operators takes two spatio-temporal objects and returns spatio-temporal objects that are intersection of them with spatio-temporal data type.

Space and spatial locus operator: Space cycle operator processing modules provide temporal space tracking operators to support spatial and spatial processing in the 
Table 2: Time stamp/interval time relation operator

Operators

tContains (stGeometry A, temporal B): Boolean

tDisjoint (stGeometry A, temporal B): Boolean

tLeftDisjoint (stGeometry A, temporal B): Boolean

tRightDisjoint (stGeometry A, temporal B): Boolean

tTouches (stGeometry A, temporal B): Boolean

tLeftTouches (stGeometry A, temporal B): Boolean

tRightTouches (stGeometry A, temporal B): Boolean

tEquals (stGeometry A, temporal B): Boolean

tContains (stGeometry A, temporal B): Boolean

tLeftContains (stGeometry A, temporal B): Boolean

tRightContains (stGeometry A, temporal B): Boolean

tDisjoint (stGeometry A, temporal B): Boolean

tLeftDisjoint (stGeometry A, temporal B): Boolean

tRightDisjoint (stGeometry A, temporal B): Boolean

tTouches (stGeometry A, temporal B): Boolean

tLeftTouches (stGeometry A, temporal B): Boolean
Explain A time $=\square$, B time $=$ )

Return specific time section $\mathrm{A}$ to include specific time section $\mathrm{A}$

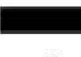

Returns whether certain time interval $\mathrm{A}$ is not met at specific time $\mathrm{B}$

$+$

Returns whether certain time B is not met before certain time interval A

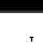

Returns whether certain time B is not met after a certain time interval A

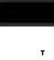

Returns whether certain time interval A contacts a certain time B

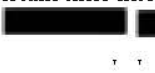

Return whether certain time zones are reached at the start time of a particular time section $\mathrm{A}$

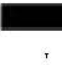

Return whether certain time zones are reached at the end of certain time interval A
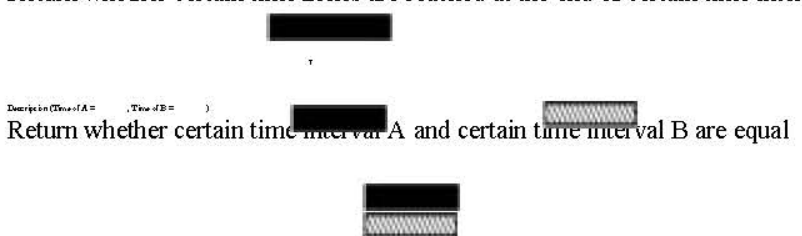

Returns whether certain time interval A contains certain time interval B

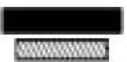

Return whether a specific time interval is included in the start time of a particular time interval A

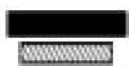

Return whether certain time interval A contains the end time of a particular time interval B

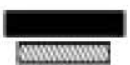

Returns whether certain time interval $\mathrm{A}$ and certain time interval $\mathrm{B}$ are not met

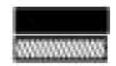

Returns whether certain time interval B is not met before certain time interval A

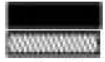

Returns whether certain time interval B is not met after a certain time interval A

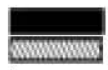

Returns whether the boundary between certain time interval A and certain time interval B is met

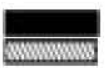

Return to the start time of a particular time interval and whether the end time of a particular time zone $B$ is reached 
Table 2: Contrine

\begin{tabular}{ll}
\hline Operators & Explain A time $=$ \\
tFight Touches (st Geometry A, temporal B): Boolean & $\begin{array}{l}\text { Retum to the end time of certain time interal A and whether the start time of a particular time zone } \\
\text { B is reached }\end{array}$
\end{tabular}

t0ver hps (stGrometry A, temporal B): Boolean

Time interwal betwe en certain time interwal A and certain time interwal B overlaps

Left Owerlaps (stGe ometry A, temporal B): Boolean

Retoms whether the start time of a particular time interwal A overlaps a certain time irterwal B

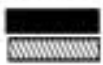

tFight Over hps (stGeometry A, temporal B): Boolean

Time interwal of the specified time interval A to retom to a partioular time interwal B

Tabl 3. Time-stamnjinterwaltime-smaksis oneretor

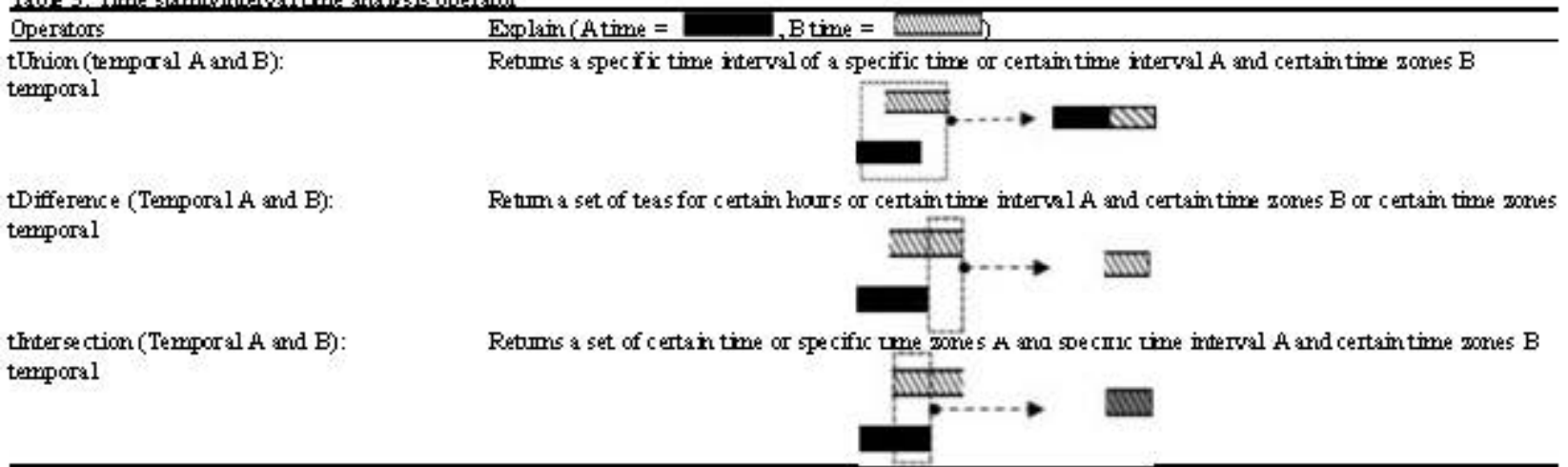

Table 4: Time stampinterwalspatio-temporalre hation operator

Operators Explain

st Equals (st Grometry A, st Grometry B): Boolean

Time interwal whe fher the same time ivterwal is equal to or equal to a certain time irterwal $\mathrm{A}$ and certain time interval A or certain time interwal B

storosses (stGeometry A, stGeometry B): Boolean

Whether a time interwal object cosses a certain time interwal in a certan time or a certain time segonert $A$ and certain time irterval B

stAppears (stGeometry A, stGeometry B): Boolean

Retom time or time interval for certain time zones A $a$ certain time irterwal A for certain time wones $\mathrm{B}$ or certain time zores

stDisappears (stGeometry A, store ometry B): Book am

Rotom whether a time interwal of a certain time or a certain time interwal of a particular time interval is loot for certain time zones A or certain time interval A

st Contains (st Grometry A, stGeometry B): Boolean

Specifi time or certain time interval A to retmn whe ther a time interwal catains a spatial or temporal space object of a particular time interwal

steftCattains (stre ometry A, stGeometry B): Boolem

Contains (stGeometry A, st Grometry B): Boolean

Return whether a time interwal of a certa in time or a certain time interval A is inchuded or whe ther a time interwal object of a particular time interval $\mathrm{B}$ or a certain time interwal is inc hded stRiffit Retirn whe ther a time $\alpha$ time zone object is inchuded in a certain time or time ivterval A for certaint time zones A hour or certain time interval B

stDigjoint (st Greometry A, stGeametry B): Boole an

A certain time a certain time irterwal of a particular time interval is notmet whether a time irterval or time interval of a particular time zone B is encountere d.

stLefDisjoint (st Grometry A, st Gre ometry B): Boole in

Retolns whe ther a time interval of a certan time or time interwal of a certsintime or a certain time interwal is rot met before a certain time interval A or certain time irterval A

strightDisjoint (stGeometry A,stGeometry B): Boolean

st Tourhes (stGre one try A, stGrometry B): Booleam

Retoms whe ther a time interval object of a certain time or a certain time interwal of a particular time interwal is not met after a certain time or a certain time interwal A

A certain time or a certim time interval of a certain time irterwal for a certain time interwal or a certain time interwal of a particular time zone B of a particular time zone B

steft Touches (stGeometry A, st Geometry B): Boolean

stFight Touches (stGe ametry A, st Grometry B): Boolesm

st Overlaps (stGeometry A, st Geometry B): Boolean

Whether a time irterval of a certain time or a certain time irterval A and a certain time interval of a partioular time interval or a time interval of a particular time zone B is met

Whether a time interval of a certain time $a$ a certain time interwal A and a time irterwal of a specific time interval or a time interval of a spe cific time interwal B hour are erc ourtered Specific time or time interwal of a particular time or time interwal of a particular time zone A or a certain time interwal of a particular time zone $B$

stLeft Over hps (stGeometry A, stGeametry B): Bolean

Time interwal when atime interval of a certain time or a specific time irterwal A over haps the time interwal of a particular time irterwal or a certan time interval B

stRight Overlaps (stGe ometry A, stGeometry B): Boolean Time interwal of whether a time irterval of a ppecific time or time interwal of a particular time interwal or a certain time interval of a particular time interval A is over lapped 
Table 5: Time Stamp/interval spatio-temporal analysis operator

\begin{tabular}{ll}
\hline Operators & Expalin \\
\hline stUnion (stGeometry A, stGeometry B): stGeometry & $\begin{array}{l}\text { Retums the union of a time interval object in a certain time or certain time interval A and certain } \\
\text { time zones B and certain time zones B } \\
\text { Retum a set of teas of a time interval object to a specific time interval or certain time interval A } \\
\text { stDifference (stGeometry A, stGeometry B): stGeometry }\end{array}$ \\
stIntersection (stGeometry A, stGeometry B): stGeometry & $\begin{array}{l}\text { Return a set of gyo objects in a specific time or time interval A and specific time interval A and } \\
\text { specific time interval B } \\
\text { Returns a spatiotemporal object that changes at a specific time or at a specific time interval A } \\
\text { with a Length L of the space-time object bounds } \\
\text { Return the distance of the space object to a certain time interval or certain time interval A and } \\
\text { certain time zones B and certain time zones }\end{array}$ \\
stDistance (stGeometry A, stGeometry B): Double &
\end{tabular}

Table 6: Spatio-temporal trajectory operator

\begin{tabular}{ll}
\hline Operators & Explain \\
stEnters (stGeometry A, stGeometry B): Boolean & $\begin{array}{l}\text { Return whether a time interval object in a certain time or a certain time interval of a certain hour } \\
\text { or a certain time interval in a certain time or a certain time interval is switched inward }\end{array}$ \\
Return whether a time interval exists within a certain time interval or a certain time interval for \\
stInsides (stGeometry A, stGeometry B): Boolean & $\begin{array}{l}\text { a particular time interval B or certain time interval B } \\
\text { Retum whether a time or time interval object in a certain time or certain time zone B of a particular } \\
\text { hour or a certain time interval is outside the internal time zone }\end{array}$ \\
& $\begin{array}{l}\text { A certain time or time interval of a certain time or a certain time interval, whether only certain time } \\
\text { zones of a particular time zone B or a certain time interval of a particular time interval are reached } \\
\text { stMeets (stGeometry A, stGeometry B): Boolean }\end{array}$ \\
& $\begin{array}{l}\text { Return whether the temporal or temporal space objects in a certain time or certain time zone B } \\
\text { or certain temporal zone B are inside and outside of a certain time interval }\end{array}$ \\
stPasses (stGeomery A, stGeometry B): Boolean &
\end{tabular}

sensor nodes. Returns true or false values for this operator. Table 6 shows the types of space and spatial trajectories.

As shown in Table 6, the spatio-temporal trajectory operator receives two spatio-temporal objects $\mathrm{A}$ and $\mathrm{B}$ as input values, performs spatio-temporal operations and returns true or false. For example, stEnter, the Space and time of space-time, accepts two spatial and spatial objects and returns whether or not a specific space object enters a certain time zone area.

\section{RESULTS AND DISCUSSION}

We implemented time relation/analysis operator, spatio-temporal relation/analysis/trajectory operator to efficiently process the spatiotemporal query entered by the user at the sensor node. Algorithm 1 shows an example of a query to perform an operation on the tRightDisjoint operators.

\section{Algorithm 1; Example of time relation operator: \\ SELECT nodeid, time, temp \\ FROM sensors \\ WHERE tRightDisjoint(tPeriod(2014/05/01 21:18:00, 2014/05/01 \\ 21:18:15), time) \\ SAMPLE PERIOD 1024}

As shown in algorithm 1, tRightDisjoint returns a sensor node ID (nodeid), time (time) and temperature (temp) not later than 21:18:00 (tPeriod, 21:18:15). Algorithm 2 shows examples of questions for performing operations on tUnion operators.

\section{Algorithm 2; Example of time analysis operator: SELECT nodeid, time, temp \\ FROM sensors \\ WHERE tContains(tUnion(tPeriod(2014/05/01 22:24:50, 2014/05/01 22:25:00), tPeriod(2014/05/01 22:24:55, 2014/05/01 22:25:10)), time) SAMPLE PERIOD 1024}

As shown in algorithm 2, the tUnion query includes a specific time interval 1 (tPeriod (2014/05/01 22:24:50, 2014/05/01 22: 25: 00)) and a specific time interval 2 sensor node ID (nodeid), time (time), temperature (temp (temp)) included in the combined time of tPeriod (2014/05/01 22: 24:55, 2014/05/01 22: 25:).

Algorithm 3 shows examples of questions to perform in relation to the computation of the stContains relationship operators.

\section{Algorithm 3; Example of spatio-temporal relation operator: \\ SELECT nodeid, temp, stLoc \\ FROM sensors \\ WHERE stContains(stPolygon(tPeriod(2014/05/01 13:47:20, 2014/05/01 13:48:00), $300300,300700,700700,700300,300300$ ), stLoc) SAMPLE PERIOD 1024}

As shown in algorithm 3, the stContains query includes a specific space-time region (stPolygon (tPeriod (2014/05/01 13: 47: 20,2014/05/01 13: 48: 00), 300300, 300 $700,700700,700,300,300,300)$ ), sensor node ID (nodeid), temperature (temp), space-time information (stLoc).

Algorithm 4 illustrates an example of a query to perform a computation of the operation of the space time analysis operator. 


\section{Algorithm 3 Example of spatio-temporal analysis operator: \\ SELECT nodeid, temp, stLoc \\ FROM sensors \\ WHERE stContains(stIntersection(stPolygon(tPeriod(2014/05/01 17:00:00,}

As shown in algorithm 4, the stIntersection query is a specific space-time region 1 (stPolygon (tPeriod (2014/05/01 17: 00: 00,201 4/05/01 19: 00: 00), 00, 0, 400, 400 $400,400 \quad 0,00)$ ) and a specific space-time region 2 (stPolygon (tPeriod (2014/05/01 18: 00: 00, 2014/05/01 20: 00: 00) (Nodeid), temperature (temp), spatio-temporal information (stLoc) included in the space-time region where the intersecting time-domain information is intersected $(100100,100500,500500,500100,100100)$. Algorithm 5 shows an example of a query to perform operations on stPasses in the spatio-temporal trajectory operator.

\author{
Algorithm 5; Example of spatio-temporal trajectory \\ operator: \\ SELECT nodeid, temp, stLoc \\ FROM sensors \\ WHERE (stPolygon (tPeriod (2014/05/01 19: 00: 00,2014/05/01 19: 10: \\ $00), 300300,300600,600600,600300,300300$ ), stLoc)
}

As shown in algorithm 5, the stPasses query includes a specific space-time region (stPolygon (tPeriod (2014/05/01 19: 00: 00,2014/05/01 19: 10: 00), 300300, 300 $600,600600,600300,300300)$ ) but returns sensor node ID (nodeid), temperature (temp), space-time information (stLoc) which go out again.

\section{CONCLUSION}

Several spatial query processing systems were investigated for efficient query processing of two-dimensional spatial sensor data on sensor networks. However, traditional spatial query systems were insufficient to process multi-dimensional sensor data because they did not support data types and operators for multi-dimensional sensor data processing. Thus, in order to address these issues, the study expanded the "Simple Features Recommendation for SQL" in the standard specification of OGC to research and develop space operators.

\section{ACKNOWLEDGEMENT}

This research was supported by the National Research Foundation of Korea (NRF) grant funded by the Korea government (MSIP) (No. 2017R1A2B4011243).

\section{REFERENCES}

Ale, R., 2012. Working with spatio-temporal data type. Proceedings of the International Conference on Archives of the Photogrammetry, Remote Sensing and Spatial Information Sciences Vol. 39, August 25- September 01, 2012, Melbourne, Australia, pp: 5-10.

Anonymous, 2011a. OpenGIS implementation specification for geographic information-simple feature access-part 1: Common architecture. Open GIS Consortium Inc, Wayland, Massachusetts.

Anonymous, 2011b. OpenGIS implementation specification for geographic information-simple feature access-part 2: SQL option. Open GIS Consortium Inc, Wayland, Massachusetts.

D'Ulizia, A., F. Ferri and P. Grifoni, 2012. Moving GeoPQL: A pictorial language towards spatio-temporal queries. GeoInformatica, 16: 357-389.

Kim, D.O., L. Liu, I.S. Shin, J.J. Kim and K.J. Han, 2013. Spatial TinyDB: A spatial sensor database system for the USN environment. Intl. J. Distrib. Sens. Networks, 9: 1-10.

Kim, S.H., D.H. Kim and H.D. Park, 2009. A tracking service of animal situation using RFID, GPS and sensor. J. Inst. Internet Broadcasting Commun., 9: 79-84.

Kim, W.I., J.J. Kim, J.G. Kim, E.Y. Park and C.Y. Kim et al., 2015. ST_SPAQL: Query language for spatio-temporal ontology. Intl. Inf. Inst. Tokyo, 18: 317-322. 